Journal of Patient-Centered

Volume 3

Issue 4 -- Cardiovascular Aging

Article 7

$11-11-2016$

\title{
Can Bedside Ultrasound Inferior Vena Cava Measurements Accurately Diagnose Congestive Heart Failure in the Emergency Department? A Clin-IQ
}

\author{
Miranda Gaskamp \\ Mark Blubaugh \\ Laine H. McCarthy \\ Dewey C. Scheid
}

Follow this and additional works at: https://aah.org/jpcrr

Part of the Cardiovascular Diseases Commons, Cardiovascular System Commons, Diagnosis Commons, and the Emergency Medicine Commons

\section{Recommended Citation}

Gaskamp M, Blubaugh M, McCarthy LH, Scheid DC. Can bedside ultrasound inferior vena cava measurements accurately diagnose congestive heart failure in the emergency department? A Clin-IQ. J Patient Cent Res Rev. 2016;3:230-4. doi: 10.17294/2330-0698.1257

Published quarterly by Midwest-based health system Advocate Aurora Health and indexed in PubMed Central, the Journal of Patient-Centered Research and Reviews (JPCRR) is an open access, peer-reviewed medical journal focused on disseminating scholarly works devoted to improving patient-centered care practices, health outcomes, and the patient experience. 


\title{
Can Bedside Ultrasound Inferior Vena Cava Measurements Accurately Diagnose Congestive Heart Failure in the Emergency Department? A Clin-IQ
}

\author{
Miranda Gaskamp, DO, ${ }^{1}$ Mark Blubaugh, DO, ${ }^{2}$ Laine H. McCarthy, MLIS, ${ }^{3}$ Dewey C. Scheid, MD, MPH \\ ${ }^{1}$ Northeastern Health Systems, Tahlequah, OK \\ ${ }^{2}$ Emergency Medicine Department, Oklahoma State University Medical Center, Tulsa, OK \\ ${ }^{3}$ Family \& Preventive Medicine Department, University of Oklahoma Health Sciences Center, Oklahoma City, OK
}

\begin{abstract}
Congestive heart failure (CHF) is a major cause of morbidity and mortality. Early diagnosis of CHF in patients presenting to the emergency department with undifferentiated dyspnea would allow clinicians to begin appropriate treatment more promptly. Current guidelines recommend B-type natriuretic peptide (BNP) levels for more accurate diagnosis of CHF in dyspneic patients. Although BNP levels are relatively inexpensive, the test is not usually performed at bedside and results may take up to an hour or more. BNP also may have a "gray zone" in which the values can neither confirm nor rule out CHF. BNP has a reported sensitivity of $87 \%$ and specificity of $74 \%$ at a cutoff of $400 \mathrm{pg} / \mathrm{ml}$. Studies investigating bedside ultrasound inferior vena cava (IVC) measurements for identifying CHF report a specificity of $84 \%$ to $96 \%$ and sensitivity values ranging from $37 \%$ to $93 \%$, depending on the study. Given that ultrasound IVC measurements are performed at bedside and results are available rapidly, it is reasonable to evaluate whether ultrasound IVC measurements obtained by appropriately trained emergency department clinicians, alone or in combination with BNP, may increase diagnostic accuracy of CHF. (J Patient Cent Res Rev. 2016;3:230-234.)
\end{abstract}

Keywords ultrasound; heart failure; inferior vena cava; pro-B-type natriuretic peptide; emergency department

\section{Clinical Question}

In adults, 18 years of age and older, who present to the emergency department (ED) with dyspnea, are inferior vena cava (IVC) measurements using bedside ultrasound as accurate as B-type natriuretic peptide (BNP) levels for identifying heart failure?

\section{Brief Answer}

Yes. Bedside ultrasound measurement of the IVC is a highly specific and rapid tool for diagnosing heart failure in the ED setting compared with BNP. BNP costs less than bedside ultrasound but is less specific and may take up to one hour or more at a clinical juncture when rapid detection of volume overload can rule in or rule out heart failure. In the studies reviewed, the specificity of the IVC measurement was

Correspondence: Laine H. McCarthy, MLIS, University of Oklahoma Health Sciences Center, 900 NE 10th Street, Oklahoma City, OK, 73118, Email: Laine-mccarthy@ouhsc.edu as good as, or better than, BNP values. Ultrasound IVC measurements may be especially useful for cases in which BNP values fall into a nondiagnostic "gray zone" range. Based on current evidence, it seems reasonable to conclude that ultrasound IVC measurements, taken by properly trained ED clinicians, alone or in combination with BNP, would likely increase accuracy of diagnosing heart failure, allow earlier initiation of appropriate treatment and potentially reduce morbidity and mortality.

Date of Literature Search: March/April 2016.

\section{Level of Evidence: B.}

\section{Search Terms}

Ultrasound, heart failure, inferior vena cava, pro-Btype natriuretic peptide, NR-PRoBNP, emergency department, diagnosis

\section{Inclusion Criteria}

Current systematic reviews, meta-analyses, cohort studies, clinical research trials investigating the 
efficacy of ultrasound measurements of the IVC for diagnosing heart failure in the ED.

\section{Exclusion Criteria}

Children less than 18 years of age, renal failure, dialysis, mechanical ventilation, trauma, abdominal surgery within two weeks, pregnancy.

\section{Summary of Issues}

In 2003, congestive heart failure (CHF) affected more than 5.7 million adults in the United States, with a lifetime incidence of 1 in 5 for individuals over age 40 years. ${ }^{1}$ Heart failure has a mortality rate of $19 \%$ and is the primary hospital discharge diagnosis for adults over age 65 . As the Baby Boomer generation continues to age, the American Heart Association estimates that 550,000 new cases of heart failure will be diagnosed each year at an estimated cost of $\$ 27$ billion annually. ${ }^{2}$

Approximately $80 \%$ of hospitalized heart failure patients are admitted from the ED. Current clinical guidelines recommend taking BNP levels to improve diagnostic accuracy of $\mathrm{CHF}$ in patients who present to the ED with undifferentiated dyspnea. BNP is a 32-amino acid polypeptide secreted by the ventricles of the heart in response to excessive stretching of heart muscle cells. A normal level $(<100 \mathrm{pg} / \mathrm{ml})$ rules out acute heart failure and an elevated level $(>500 \mathrm{pg} / \mathrm{ml})$ in the absence of renal failure can be diagnostic. ${ }^{2}$ A study evaluating the efficacy of BNP in correctly diagnosing CHF reported a sensitivity of $96 \%$ and specificity of $26 \%$ at a cutoff level of $100 \mathrm{pg} / \mathrm{ml}$; at a cutoff level of $400 \mathrm{pg} / \mathrm{ml}$, BNP had a sensitivity of $87 \%$ and specificity of $74 \% .{ }^{3}$ However, BNP levels can have a "gray zone" in which the values neither confirm nor rule out CHF. In addition, BNP levels are not generally done at the bedside and results can take up to an hour at a time when rapid recognition of volume overload is critical for early treatment. ${ }^{1}$

Ultrasound IVC measurements, performed at bedside in the ED, are rapid and potentially more specific for diagnosing CHF. Measuring the IVC also may be more accurate for identifying alternative causes of dyspnea, such as pneumonia, chronic obstructive pulmonary disease (COPD), noncardiogenic pulmonary edema and lung cancer. ${ }^{4}$ This brief report examines the evidence for using bedside ultrasound IVC, alone or in addition to BNP, for accurately identifying heart failure versus other causes of dyspnea.

\section{Summary of the Evidence}

Studies show that bedside ultrasound measurements of the IVC correlate with central venous pressure and response to fluid administration. A small IVC $(<2$ $\mathrm{cm}$ in diameter), with collapse greater than $50 \%$ on inspiration, corresponds to a central venous pressure $<$ $10 \mathrm{~cm}$ of water, and vice versa. ${ }^{4}$ Published guidelines by the American Society of Echocardiography support the evaluation of IVC size and collapsibility in the assessment of central venous pressure. ${ }^{1}$ Studies in patients with heart failure used IVC measurements to estimate elevated cardiac filling pressures. ${ }^{5}$

In 2009, Blehar et al. used IVC measurements to identify CHF in a convenience sample of 46 adults (age $\geq 18$ years) who presented at the ED with the complaint of dyspnea. ${ }^{6}$ Patients who had received a liver transplant, were on mechanical ventilation, taking diuretics, bronchodilators or vasoactive medications, or who were pregnant were excluded. Study participants received ultrasound IVC measurements using a $3.5 \mathrm{MHz}$ curvilinear probe at bedside prior to any interventions. Minimum diameter during inspiration and maximum diameter during expiration were measured. Variation was calculated as the difference between maximum and minimum divided by the maximum. Patients with CHF should have less variation in IVC diameter because volume overload does not allow as much IVC collapse as normally occurs during inspiration. Two physicians, blinded to the ultrasound results, used a standardized chart review to retrospectively determine the final diagnosis as $\mathrm{CHF}$ or an alternate diagnosis. ${ }^{6}$

The study showed that respiratory variation of the IVC was smaller in patients with CHF $(9.6 \%)$ than without CHF (46\%). ${ }^{6}$ At the optimum cutoff value of $15 \%$ variation or less of the IVC diameter, the sensitivity was $93 \%$ (95\% confidence interval [CI]: 76-99) and the specificity was $84 \%$ (95\% CI: $77-88$ ) for the diagnosis of CHF (Table 1). Four participants who presented with shortness of breath and BNP measurements $>100 \mathrm{pg} / \mathrm{ml}$ were correctly identified by ultrasound IVC measurements as non-CHF. The main limitations of this study were its small study size and the lack of echocardiogram to rule out confounding factors like tricuspid regurgitation. ${ }^{6}$

In 2012, Miller et al. determined the sensitivity and specificity of a caval index for diagnosing acute heart failure in 89 adults (age $\geq 50$ years), who 
Table 1. Sensitivity and Specificity of IVC Measurements for Diagnosing Congestive Heart Failure

\begin{tabular}{lccc}
\hline Study (number of subjects) & $\begin{array}{c}\text { IVC-Cl diameter } \\
\text { variation cutoff }\end{array}$ & $\begin{array}{c}\text { Sensitivity } \\
\mathbf{( 9 5 \% ~ C l )}\end{array}$ & $\begin{array}{c}\text { Specificity } \\
\mathbf{( 9 5 \% ~ C l )}\end{array}$ \\
\hline Blehar et al. ${ }^{6}(\mathrm{~N}=46)$ & $<15 \%$ & $93 \%(76-99)$ & $84 \%(77-88)$ \\
Miller et al. ${ }^{5}(\mathrm{~N}=89)$ & $<15 \%$ & $37 \%(22-55)$ & $96 \%(86-99)$ \\
Anderson et al. ${ }^{1}(\mathrm{~N}=101)$ & $<33 \%$ & $80 \%(63-91)$ & $81 \%(68-90)$ \\
Yamanoğlu et al. ${ }^{7}(\mathrm{~N}=74)$ & $<20 \%$ & $52 \%(38-67)$ & $86 \%(77-95)$ \\
\hline
\end{tabular}

$\mathrm{Cl}$, confidence interval; IVC-Cl, inferior vena cava collapsibility index.

presented at the ED with undifferentiated dyspnea. ${ }^{5}$ Patients were excluded if they required mechanical ventilation, experienced recent trauma, had abdominal surgery within the past two weeks, had known portal hypertension or were pregnant. Two physicians took ultrasound measurements with a cardiac phased-array probe of the maximum IVC during expiration and the minimum IVC during inspiration over three respiratory cycles prior to any interventions. A caval index was calculated as the difference between the IVC during expiration and inspiration divided by the IVC during expiration multiplied by 100 (IVCe - IVCi)/(IVCe $\times$ 100). This study found that a caval index of $<15 \%$ had a sensitivity of $37 \%(95 \%$ CI: $22-55)$ and a specificity of $96 \%$ (95\% CI: $86-99$ ); at a caval index of $33 \%$, the sensitivity was $80 \%$ (95\% CI: 63-91) and the specificity was $81 \%$ (95\% CI: 68-90) (Table 1). A limitation of this study for the purposes of this review is that the diagnostic characteristics of BNP were reported but not directly compared to ultrasound measurements. The main limitation of this study was its small sample size. ${ }^{5}$

In a 2013 study, Anderson and colleagues investigated the accuracy of IVC collapsibility index (IVC-CI), left ventricular ejection fraction (LVEF) and lung B-lines measured by ultrasound for diagnosing acutely decompensated heart failure in the ED. ${ }^{1}$ The prospective convenience cohort study enrolled 101 adults (age $\geq 18$ years) who presented at the ED with acute dyspnea. BNP levels were ordered for all study participants. Clinical investigators, who were emergency ultrasound fellowship-trained and blinded to all clinical data, performed the ultrasound measurements of the IVC maximum and minimum, LVEF and lung B-lines. These measurements were compared to a final diagnosis as determined by chart review conducted by two emergency physicians blinded to the ultrasound results. ${ }^{1}$

At a cutoff point of $20 \%$, the IVC-CI had a sensitivity of $52 \%$ (95\% CI: $38-67)$ and a specificity of $86 \%$ (95\% CI: 77-95) for accurately diagnosing acutely decompensated heart failure (Table 1), similar to the specificity of BNP levels $>500 \mathrm{pg} / \mathrm{ml}(83 \%$ [95\% CI: 67-92]). ${ }^{1}$ When IVC-CI measurements were combined with both LVEF and lung B-lines, the specificity went up to $100 \%$ (95\% CI: 92-100). These results suggest that ultrasound tests measuring IVC-CI, LVEF and lung B-lines may be the most accurate and effective means of diagnosing heart failure at bedside. This study was limited by its smaller size and possible selection bias from the convenience sampling methodology. There may have been some misclassifications based on the retrospective chart review and potential bias introduced by other test values recorded in charts from prior non-ED encounters. For example, a smaller IVCCI variability may have been recorded because a low ejection fraction was already noted in the chart. ${ }^{1}$

In a 2014 prospective, observational study, Yamanoğlu et al. used IVC diameter measurements to differentiate between dyspnea of cardiac origin and pulmonary dyspnea. ${ }^{7}$ Inclusion criteria were met by 74 elderly adults (mean age: 72.8 years) who presented to the ED during the study time window. Patients with severe tricuspid regurgitation, cardiac tamponade, aortic dissection, abdominal surgery within the preceding two weeks, or those who had cardiopulmonary arrest, had been intubated or were pregnant were excluded. Of the 74 enrolled patients, 32 received a final diagnosis of cardiac-associated dyspnea. B-mode IVC diameter greater than $9 \mathrm{~mm}$ during inspiration had the greatest sensitivity $(84.4 \%)$ and specificity 
(92.9\%) for identifying dyspnea of cardiac origin; IVC-CI was similar, with a sensitivity of $84.4 \%$ and specificity $90.5 \%$ (Table 1 ). This study concluded that ultrasound IVC measurements were "rapid, readily available, inexpensive, reproducible techniques" for differentiating dyspnea due to cardiac etiology (acute heart failure) from dyspnea of pulmonary origin. ${ }^{7}$

Ultrasound technology is a common diagnostic tool in the $\mathrm{ED}^{8}$ and is a required competency in all U.S. Accreditation Council for Graduate Medical Education emergency medicine programs. ${ }^{7,9}$ Bedside ultrasound IVC measurement shows promise for use in appropriately diagnosing CHF. Diagnostic accuracy would be improved with standardization of the location of IVC diameter measurement and probe orientation (i.e. long vs short axis), which may vary depending on training and experience of ED physicians. ${ }^{9}$ Reports have documented the benefit of training targeting the specific skills required for accurate performance and reading ultrasound test results in the ED. ${ }^{10}$

\section{Limitations}

None of the studies reviewed in this report were randomized controlled trials of efficacy. However, a minority of studies of diagnostic tests meet this standard. The four studies reported ${ }^{1,5-7}$ were prospective. In three of the four, ${ }^{1,5,6}$ there was an independent blind comparison with reference standards. All of the studies used convenience samples, which increases the risk of selection bias. However, the papers reviewed included a broad spectrum of patients with characteristics generalizable to the ED population. Small sample sizes in each of the papers cited resulted in moderately wide confidence intervals. Larger studies or a metaanalysis combining the data of several studies could improve the precision of the estimates of sensitivity and specificity of IVC-CI. However, a meta-analysis may not be feasible due to differences in ultrasound technique, study protocols and other potential causes of study heterogeneity. Randomized controlled trials are needed to evaluate the efficacy of bedside ultrasound compared to BNP or the combination of BNP and bedside ultrasound in the management of CHF.

\section{Conclusions}

Bedside ultrasound inferior vena cava measurements in the presence of undifferentiated dyspnea in the emergency department may be as helpful as, and more rapid than, B-type natriuretic peptide values for diagnosing congestive heart failure. In the studies reviewed, IVC-CI (ranging from 15\% to 55.5\%) had a high specificity $(84 \%-96 \%)$ for identifying heart failure, which would allow ED physicians to avoid prematurely treating patients who are not experiencing $\mathrm{CHF}$ and revise their plan while collecting additional data. In all instances, the specificity of ultrasound IVC was as good as or better than BNP values and may help achieve appropriate diagnosis for patients in which BNP values fall in the "gray zone." Bedside ultrasound IVC assessment also may be useful for the early recognition of other causes of dyspnea and comorbid conditions such as COPD and other respiratory illnesses - that are increasing in prevalence among elderly patients. Ultrasound IVC allows physicians to begin treating even unstable patients earlier, before results from more traditional tests like BNP become available. ${ }^{1}$

Now that the accuracy of bedside ultrasound has been established, the next step would be undertaking studies that evaluate the accuracy of ED physician-obtained IVC measurements by ultrasound. Ideally, an ED physician should be able to accurately and efficiently assess noncollapsibility, as can be done for collapsibility, by fluid resuscitation. If randomized controlled trials demonstrate the efficacy of bedside ultrasound compared to BNP in the management of CHF, costeffectiveness studies comparing diagnostic methods and strategies should be undertaken to determine whether the additional cost of ultrasound ( $\sim 264$ vs $\sim \$ 88$ for BNP, per Healthcare Bluebook mobile app) provides significant improvements in diagnostic accuracy, morbidity, mortality and quality of life.

\section{Patient-Friendly Recap}

- Heart failure is often diagnosed in the emergency department using a specific blood test that takes up to an hour to yield results.

- Bedside ultrasound technology, a common diagnostic tool used in emergency departments, has shown promise in measuring heart function.

- The authors reviewed findings from several published studies and concluded that measuring a patient's inferior vena cava with ultrasound may help quickly and accurately diagnose congestive heart failure. 


\section{Conflicts of interest}

None.

\section{Funding Sources}

Laine McCarthy is by supported by Oklahoma Shared Clinical \& Translational Resources and a grant (NIGMS U54GM104938) by the National Institute of General Medical Sciences, National Institutes of Health.

\section{Acknowledgments}

The authors thank Frederick C. Miller, PhD, Bruna M. Varalli-Claypool, MHS, PA-C, James Brand, MD, William Wylie, DO, and Jessica Brockhaus, BA, for reading and commenting on this manuscript.

\section{References}

1. Anderson KL, Jeng KY, Fields JM, Panebianco NL, Dean AJ. Diagnosing heart failure among acutely dyspneic patients with cardiac, inferior vena cava, and lung ultrasonography. Am J Emerg Med. 2013;31:1208-44. CrossRef

2. Silvers SM, Howell JM, Kosowsky JM, Rokos IC, Jagoda AS; American College of Emergency Physicians Clinical policy: critical issues in the evaluation and management of adult patients presenting to the emergency department with acute heart failure. Ann Emerg Med. 2007;49:627-69. CrossRef
3. Parab R, Vasudevan A, Brensilver J, Gitler B. Utility of brain natriuretic peptide as a diagnostic tool for congestive heart failure in the elderly. Crit Pathw Cardiol. 2005;4:140-4. CrossRef

4. Russell FM, Ehrman RR, Cosby K, et al. Diagnosing acute heart failure in patients with undifferentiated dyspnea: a lung and cardiac ultrasound (LuCUS) protocol. Acad Emerg Med. 2015;22:182-91. CrossRef

5. Miller JB, Sen A, Strote SR, et al. Inferior vena cava assessment in the bedside diagnosis of acute heart failure. Am J Emerg Med. 2012;30:778-83. CrossRef

6. Blehar DJ, Dickman E, Gaspari R. Identification of congestive heart failure via respiratory variation of inferior vena cava diameter. Am J Emerg Med. 2007;27:71-5. CrossRef

7. Yamanoğlu A, Yamanoğlu NG, Parlak İ, et al. The role of inferior vena cava diameter in the differential diagnosis of dyspneic patients; best sonographic measurement method? Am J Emerg Med. 2015;33:396-401. CrossRef

8. Perera P, Mailhot T, Riley D, Mandavia D. The RUSH exam: Rapid Ultrasound in SHock in the evaluation of the critically ill. Emerg Med Clin North Am. 2010;28:29-56. CrossRef

9. Wallace DJ, Allison M, Stone MB. Inferior vena cava percentage collapse during respiration is affected by the sampling location: an ultrasound study in healthy volunteers. Acad Emerg Med. 2010;17:96-9. CrossRef

10. Ehrman RR, Frances MR, Ansari AH, et al. Can emergency physicians diagnose and correctly classify diastolic dysfunction using bedside echocardiography? Am J Emerg Med. 2015;33:1178-83. CrossRef

(C) 2016 Aurora Health Care, Inc. 